\title{
ORIGINAL
}

\section{PREPARACIÓN DEL PERSONAL SANITARIO EN UN ÁREA DE SALUD DE ASTURIAS ALTAMENTE INDUSTRIALIZADA PARA RESPONDER ANTE UN DESASTRE INDUSTRIAL (*)}

\section{Rafael Castro Delgado, Pedro Arcos González y Tatiana Cuartas Álvarez.}

Unidad de Investigación en Emergencias y Desastres. Departamento de Medicina. Universidad de Oviedo. (*) Proyecto financiado por el II Plan Regional de Investigación del Principado de Asturias (proyecto PP-AMB-98-02).

\section{RESUMEN}

Fundamento: Para responder a los accidentes industriales, de cara a minimizar sus consecuencias, es necesaria una correcta preparación del personal sanitario que trabaja en zonas de riesgo. El objetivo de este estudio es establecer el grado de percepción subjetiva que tienen los profesionales sanitarios sobre su preparación para actuar en un accidente industrial con múltiples víctimas, así como su conocimiento sobre los riesgos industriales propios de la zona.

Métodos: Se diseñó un cuestionario para medir cinco dimensiones relacionadas con la respuesta ante accidentes industriales (conocimiento técnicos, conocimientos del Area, habilidades prácticas, actitudes y formación). La muestra estaba formada por todos los médicos y personal de enfermería de Atención Primaria del Área y del Servicio de Urgencias del hospital de referencia.

Resultados: La tasa de respuesta fue del $61,2 \%$. Los resultados en las cuestiones de cada dimensión, con un valor máximo de 4 , fueron: Conocimientos técnicos $(\mu=1,3 ; \delta=0,81)$; Conocimientos del Área $(\mu=0,75 ; \mathrm{d}=0,96)$; Habilidades prácticas $(\mu=0,9 ; \delta=0,8)$; Actitudes $(\mu=3,25 ; \delta=0,73)$; y Formación $(\mu=0,75 ; \delta=0,88)$. Obtienen mejores puntuaciones los trabajadores del servicio de urgencias hospitalario y aquellos con contratos indefinidos o temporales, por encima de los trabajadores con contrato fijo.

Conclusiones: Existe un déficit de información y de formación entre los profesionales sanitarios que contrasta con el interés mostrado por subsanar estas carencias. Es necesario establecer un programa de información y formación básica para responder ante desastres industriales en nuestra Área de Salud.

Palabras clave: Desastre industrial. Medicina de emergencia. Desastres químicos. Planificación en desastres. Formación médica continuada. Apoyo a la formación profesional.

Correspondencia:

Dr. Rafael Castro Delgado

Unidad de Investigación en Emergencias y Desastres

Área de Medicina Preventiva y Salud Pública

Facultad de Medicina. C/ Julián Clavería, 6. Oviedo.

Correo electrónico: rafacast@netcom.atodavela.com

\section{ABSTRACT}

\section{Healthcare Personnal Training in a Healthcare District to Respond to an Industrial Disaster. Asturias, Spain}

Background: In order to provide a response to industrial accidents with a view to minimizing their consequences, the healthcare personnel working in areas at risk must be properly trained. This study is aimed at determining the degree of subjective perception that healthcare professional have with regard to how well-prepared they are to take action in response to an industrial accident resulting in a large number of injuries, as well as their knowledge of industrial hazards inherent to the area.

Methods: A questionnaire was designed to measure five dimensions related to the response to industrial accidents (technica knowledge, knowledge of the Area, practical skills, attitudes an training). The sample was comprised of all of the Primary Care physicians and nursing staff in the District and from the Emergency Department at the related hospital.

Results: The response rate was $61.2 \%$. The results for the questions related to each dimension, on a maximum scale of 4 , were: Technical skills $(\mu=1.3 ; \delta=0.81)$; Knowledge of the District $(\mu=0.75 ; \delta=0.96)$; Practical skills $(\mu=0.9 ; \delta=0.8)$; Attitudes $(\mu=3.25 ; \delta=0.73)$ and Training $(\mu=0.75 ; \delta=0.88)$. Those working in hospital emergency departments and those hired under indefinite or temporary contracts scored higher than those employed under permanent contracts.

Conclusions: Information and training is lacking among these healthcare professionals, contrasting with the interest shown in correcting these lacks. It is necessary for a basic training and information program to be set up in order to deal with industrial disasters in our Healthcare District.

Key words: Industrial disaster. Emergency medicine. Disaster planning. Training support. 


\section{INTRODUCCIÓN}

Accidentes industriales como el ocurrido en Toulouse (Francia) en Septiembre de 2001 en que la explosión en una fábrica de fertilizantes causó 30 muertos, 9000 heridos y más de 800 hospitalizados muestran que, si bien el desarrollo industrial es uno de los principales factores de mejora de la calidad de vida de las sociedades, también implica un riesgo que en determinadas circunstancias se traduce en accidentes que desbordan la capacidad de respuesta de la estructura sanitaria $^{1,2}$ e implican un problema importante de salud pública. Por ello, en zonas con fuerte concentración industrial cercana a poblaciones $^{3-, 5}$ es necesario que el sistema sanitario haya desarrollado planes de respuesta frente a accidentes industriales ${ }^{6-9}$ y que sus profesionales adquieran los conocimientos mínimos para la adecuada asistencia sanitaria a los afectados ${ }^{10}$.

En el Principado de Asturias, especialmente en el Área de Salud III, ha existido tradicionalmente un importante tejido industrial que ha influido de forma importante en su demografía y vías de comunicación ${ }^{11}$, así como en la organización de su estructura sanitaria $^{12}$, que depende principalmente de las características y dispersión de la población. Esta área, con 160083 habitantes sobre una superficie total de $482.5 \mathrm{Km}^{2}$ tiene como núcleos de población más importantes Avilés (80870 habitantes), Piedras Blancas (8869), Las Vegas (8250), Luanco (4948), Salinas (4852) y Pravia $(4783)^{11}$.

Estudios previos realizados ${ }^{13}$ han mostrado que este área tiene una importante concentración de instalaciones industriales de riesgo ${ }^{14}$ próximas a núcleos urbanos con alta densidad de población lo que justificaría, por un lado, una adecuación de los recursos sanitarios de respuesta a una emergencia industrial con afectación de la población general $^{15}$, tanto a nivel organizativo como de formación de los profesionales sanitarios de Atención Primaria ${ }^{16-18}$ y Especializada ${ }^{19-21}$.
En la actualidad existen dos empresas afectadas por el Real Decreto 886/1988 sobre prevención de accidentes mayores en determinadas actividades industriales ${ }^{22}$, por lo que la Autoridad Competente regional, en este caso el 112, ha desarrollado los correspondientes planes de emergencia exterior, si bien es de destacar que con la entrada en vigor del Real Decreto 1254/1999 por el que se aprueban las medidas de control de los riesgos inherentes a los accidentes graves en los que intervengan sustancias peligrosas ${ }^{23}$, que deroga el anterior, más empresas del área se verán afectadas.

El objetivo de este estudio es establecer el grado de percepción subjetiva que tienen los profesionales sanitarios sobre su preparación para responder a un accidente industrial con múltiples víctimas, así como su conocimiento sobre los riesgos industriales propios de la zona, algo que sería un importante indicador sobre el grado de implantación de los planes de emergencia entre el personal sanitario.

La hipótesis planteada es que tanto esta percepción subjetiva como los conocimientos sobre los riesgos industriales locales son insuficientes como para lograr una adecuada respuesta sanitaria, y que el grado de autovaloración se relaciona con las características profesionales del personal sanitario.

\section{MATERIAL Y MÉTODOS}

Para lograr los objetivos planteados se realizó una encuesta de autovaloración subjetiva al personal médico y de enfermería potencialmente implicado en la respuesta sanitaria a un desastre industrial. El periodo de realización fue entre Enero y Junio de 2000. La población considerada universo estadístico fueron los 227 médicos y enfermeras/os que trabajan en el Servicio de Urgencias del hospital de referencia del área (Hospital San Agustín de Avilés), así como en el ámbito de la Atención Primaria en todo 
el Área III. En total había 19 médicos y 18 enfermeros/as en el servicio de urgencias del Hospital San Agustín, así como 87 médicos y 103 enfermeros/as en Atención Primaria. Los primeros se incluyeron por ser quienes atenderían las víctimas que precisasen atención hospitalaria en el área y los segundos por poder tener que atender afectados en el lugar del accidente.

Tras un primer diseño del cuestionario, que fue pilotado con 20 profesionales sanitarios de Centros de Salud del área tomados al azar, este se analizó y se modificó para obtener el cuestionario finalmente empleado (ver Anexo 1) que constaba de 27 preguntas agrupadas en las cinco dimensiones que se pretendían medir (conocimientos técnicos, conocimientos del área, habilidades prácticas, actitudes y formación). La consistencia interna de las diferentes dimensiones del cuestionario se analizó mediante el coeficiente alfa de Cronbach $^{24}$.

Para la realización de las encuestas se programaron reuniones previas en cada centro de salud con los profesionales sanitarios. A los 15 días de esta reunión se distribuyeron los cuestionarios que se recogieron en los 15 días siguientes. El tratamiento estadístico de los resultados se realizó con el programa SPSS versión 8.0.0. En la comparación de variables independientes cualitativas dicotómicas con variables dependientes se usó una prueba t de Student y en la comparación de variables cualitativas con más de dos categorías con las variables dependientes se usó una prueba ANOVA. En la comparación variables cuantitativas se usó el coeficiente de correlación de Pearson. En todos los casos se consideraron significativas las diferencias halladas con una confianza del $95 \%$.

\section{RESULTADOS}

El universo estadístico estaba formado por 227 personas, de las que 139 (61,2\%) contestaron finalmente a la encuesta. Del total, 106 eran médicos, de los que contestaron $64(60,3 \%)$ y 121 eran enfermeras/os, de los que contestaron 75 (61,9\%). De los 19 médicos del servicio de Urgencias del Hospital San Agustín contestaron 14 (73,6\%), y de las 18 enfermeras/os contestaron 13 $(72,2 \%)$. En el caso de los profesionales de atención primaria contestaron 50 médicos $(57 \%)$ y 65 enfermeras/os (63\%).

La edad media de los encuestados fue de 40,2 años (=7,8; rango 28-65). En el caso de los años trabajados en el área, la media fue de 9,6 ( =6,8; rango 0,8-34); la media de años que han estado trabajando en asistencia sanitaria urgente fue de $10,1 \quad(=7,0$; rango 0,5-38). De los encuestados, 55 (39,6\%) fueron hombres y $84(60,4 \%)$ mujeres. En cuanto a las categorías profesionales, $31(22,3 \%)$ fueron médicos, $30(21,6 \%)$ médicos con formación MIR, 3 (2,2\%) médicos coordinadores de equipo, $1(0,7 \%)$ médico coordinador de equipo con formación MIR, 66 $(47,5 \%)$ enfermeras/os y $8(5,8 \%)$ enfermeras/os coordinadores de enfermería. Del total de encuestados 64 (46\%) fueron médicos y $75(54 \%)$ personal de enfermería. En cuanto al tipo de contrato, $84(60,5 \%)$ eran personal fijo, 32 (23\%) indefinido y 23 $(16,5 \%)$ temporal.

Los valores del coeficiente alfa de Cronbach para cada dimensión fueron los siguientes: 0,81 para la dimensión Conocimientos técnicos; 0,68 para Conocimientos del área; 0,78 para Habilidades prácticas; 0,67 para Actitudes; y 0,73 para Formación.

Los resultados medios de las puntuaciones obtenidas en cada dimensión han sido: a) Conocimientos técnicos, sobre un máximo de 28 puntos, $(\mu=9,15 ; \delta=4)$; b) Conocimientos del Área, sobre un máximo de 22 puntos $(\mu=4,48 ; \delta=3,79) ; c)$ Habilidades prácticas, sobre un máximo de 20 puntos $(\mu=4,51 ; \delta=$ 2,96; d) Actitudes, sobre un máximo de 12 puntos $(\mu=9,72 ; \delta=2,04) ;$ y e) Formación, sobre un máximo de 12 puntos $(\mu=2,22$, $\delta=2,15$ ). Las puntuaciones medias obtenidas 
Tabla 1

Resultados completos de la dimensión Conocimientos Técnicos y Conocimientos del Área

\begin{tabular}{|c|c|c|c|c|c|c|}
\hline $\begin{array}{l}\text { Conocimientos } \\
\text { técnicos }\end{array}$ & $\begin{array}{c}0 \\
\text { n (\%) }\end{array}$ & $\begin{array}{c}1 \\
\text { n (\%) }\end{array}$ & $\begin{array}{c}2 \\
\text { n (\%) }\end{array}$ & $\begin{array}{c}3 \\
\text { n (\%) }\end{array}$ & $\begin{array}{c}4 \\
\text { n (\%) }\end{array}$ & $\begin{array}{l}\text { Total } \\
\text { n (\%) }\end{array}$ \\
\hline Pregunta 1 & $16(11,5)$ & $99(71,2)$ & $18(12,9)$ & $5(3,6)$ & $1(0,7)$ & $139(100)$ \\
\hline Pregunta 2 & $29(20,9)$ & $96(69,1)$ & $12(8,6)$ & $1(0,7)$ & $1(0,7)$ & $139(100)$ \\
\hline Pregunta 3 & $48(34,5)$ & $38(27,3)$ & $37(26,6)$ & $10(7,2)$ & $6(4,3)$ & $139(100)$ \\
\hline Pregunta 4 & $48(34,5)$ & $76(54,7)$ & $11(7,9)$ & $2(1,4)$ & $1(0,7)$ & $138(99,3)$ \\
\hline Pregunta 5 & $31(22,3)$ & $91(65,5)$ & $14(10,1)$ & $2(1,4)$ & $1(0,7)$ & $139(100)$ \\
\hline Pregunta 6 & $3(2,2)$ & $11(7,9)$ & $65(46,8)$ & $40(28,8)$ & $20(14,4)$ & $139(100)$ \\
\hline \multirow[t]{2}{*}{ Pregunta 7} & $6(4,3)$ & $55(39,6)$ & $46(33,1)$ & $19(13,7)$ & $11(7,9)$ & $137(98,6)$ \\
\hline & Recursos & Inesperado & Muchos & Daños & Actuaciones & \\
\hline \multirow[t]{3}{*}{ Pregunta 8} & desbordados & $\mathrm{y} / \mathrm{o}$ & Afectados & Medio & especiales & \\
\hline & & urgente & & ambiente & & \\
\hline & $23(16,5)$ & $37(26,6)$ & $67(48,2)$ & $5(3,6)$ & $18(12,9)$ & \\
\hline Conocimientos & $\mathbf{0}$ & 1 & 2 & 3 & 4 & Total \\
\hline del área & n (\%) & n (\%) & n (\%) & n (\%) & n (\%) & n (\%) \\
\hline Pregunta 1 & $70(50,4)$ & $5(3,6)$ & $31(22,3)$ & $25(18)$ & $6(4,3)$ & $137(98,6)$ \\
\hline Pregunta 2 & $49(35,3)$ & $56(40,3)$ & $23(16,5)$ & $10(7,2)$ & $1(0,7)$ & $139(100)$ \\
\hline Pregunta 3 & $76(54,7)$ & $35(25,2)$ & $25(18)$ & & & $136(97,8)$ \\
\hline Pregunta 4 & $45(32,4)$ & $79(56,8)$ & $9(6,5)$ & $4(2,9)$ & $2(1,4)$ & $139(100)$ \\
\hline Pregunta 6 & $115(82,7)$ & $1(0,7)$ & $4(2,9)$ & $1(0,7)$ & $18(12,9)$ & $139(100)$ \\
\hline Pregunta 7 & $109(78,4)$ & $25(18)$ & $3(2,2)$ & $0(0)$ & $1(0,7)$ & $138(99,3)$ \\
\hline \multirow[t]{2}{*}{ Pregunta 5} & Natural & $\begin{array}{c}\text { Concentraciones } \\
\text { humanas }\end{array}$ & $\begin{array}{l}\text { Tránsito } \\
\text { Múltiples } \\
\text { víctimas }\end{array}$ & $\begin{array}{c}\text { Mercancías } \\
\text { peligrosas }\end{array}$ & $\begin{array}{l}\text { Accidente } \\
\text { mayor } \\
\text { industrial }\end{array}$ & Otros \\
\hline & $18(12,9)$ & $9(6,5)$ & $110(79,1)$ & $46(33,1)$ & $98(70,5)$ & $2(1,5)$ \\
\hline \multirow[t]{2}{*}{ Pregunta 5 (Otros) } & Naufragio & Avión & & & & \\
\hline & $1(0,7)$ & $1(0,7)$ & & & & \\
\hline
\end{tabular}

en las preguntas de cada dimensión, sobre un máximo de 4, han sido: a) Conocimientos técnicos $(\mu=1,3 ; \delta=0,81)$; b) Conocimientos del Área $(\mu=0,75 ; \delta=0,96)$; c) Habilidades prácticas $(\mu=0,9 ; \delta=0,8 ; d)$ Actitudes $(\mu=3,25 ; \delta=0,73)$; y e) Formación $(\mu=0,75$; $\delta=0,88)$.

Con respecto a los resultados obtenidos en la dimensión de conocimientos técnicos, han obtenido puntuaciones globales significativamente mayores los profesionales que trabajan en centros de salud o consultorios con industrias en su zona de influencia ( $\mathrm{p}=0,003)$, los que tienen contratos temporales $(\mathrm{p}=0,003)$ y los trabajadores del servicio de urgencias hospitalario ( $\mathrm{p}=0,000)$; además existe una correlación inversa y significativa $(\mathrm{r}=-0,232 ; \mathrm{p}=0,007)$ entre edad y conocimientos técnicos. Los resultados completos de la dimensión conocimientos técnicos se muestran en la tabla 1.

En el caso de los conocimientos del área, las puntuaciones totales obtenidas han sido significativamente mejores entre los encues- 
Tabla 2

Resultados completos de las dimensiones Habilidades Prácticas, Actitudes y Formación

\begin{tabular}{|c|c|c|c|c|c|c|}
\hline $\begin{array}{c}\text { Habilidades } \\
\text { prácticas }\end{array}$ & $\begin{array}{c}0 \\
\text { n (\%) }\end{array}$ & $\begin{array}{c}1 \\
\text { n (\%) }\end{array}$ & $\begin{array}{c}2 \\
\text { n (\%) }\end{array}$ & $\begin{array}{c}3 \\
\text { n (\%) }\end{array}$ & $\begin{array}{c}4 \\
\text { n (\%) }\end{array}$ & $\begin{array}{l}\text { Total } \\
\text { n (\%) }\end{array}$ \\
\hline Pregunta 1 & $18(12,9)$ & $65(46,8)$ & $39(28,1)$ & $12(8,6)$ & $3(2,2)$ & $137(98,6)$ \\
\hline Pregunta 2 & $76(54,7)$ & $51(36,7)$ & $11(7,9)$ & $0(0)$ & $0(0)$ & $138(99,3)$ \\
\hline Pregunta 3 & $51(36,7)$ & $49(35,3)$ & $28(20,1)$ & $8(5,8)$ & $3(2,2)$ & $139(100)$ \\
\hline Pregunta 4 & $68(48,9)$ & $60(43,2)$ & $8(5,8)$ & $2(1,4)$ & $0(0)$ & $138(99,3)$ \\
\hline Pregunta 5 & $32(23)$ & $76(54,7)$ & $23(16,5)$ & $5(3,6)$ & $1(0,7)$ & $137(98,6)$ \\
\hline \multirow{4}{*}{ Pregunta 6 (Otros) } & Lugar & $\begin{array}{c}\text { Tipo de } \\
\text { accidente/ } \\
\text { sustancia }\end{array}$ & $\begin{array}{l}\text { Número } \\
\text { afectados }\end{array}$ & $\begin{array}{l}\text { Situación } \\
\text { clínica }\end{array}$ & Otros & \\
\hline & $52(37,4)$ & $90(64,7)$ & $88(63,3)$ & $\begin{array}{l}43(30,9) \\
\text { Recursos }\end{array}$ & $35(25,2)$ & \\
\hline & Accesos & $\begin{array}{l}\text { Efecto } \\
\text { dominó }\end{array}$ & $\begin{array}{l}\text { Zona de } \\
\text { influencia }\end{array}$ & $\begin{array}{c}\text { Necesarios } \\
\text { y/o disponibles }\end{array}$ & & \\
\hline & $2(1,4)$ & $7(5)$ & $4(2,9)$ & $21(15,1)$ & & \\
\hline Actitudes & $\begin{array}{c}0 \\
\text { n (\%) }\end{array}$ & $\begin{array}{c}1 \\
\text { n (\%) }\end{array}$ & $\begin{array}{c}2 \\
\text { n (\%) }\end{array}$ & $\begin{array}{c}3 \\
\text { n (\%) }\end{array}$ & $\begin{array}{c}4 \\
\text { n (\%) }\end{array}$ & $\begin{array}{l}\text { Total } \\
\text { n (\%) }\end{array}$ \\
\hline Pregunta 1 & $1(0,7)$ & $14(10,1)$ & $38(27,3)$ & $54(38,8)$ & $29(20,9)$ & $136(97,8)$ \\
\hline Pregunta 2 & $2(1,4)$ & $0(0)$ & $2(1,4)$ & $44(31,7)$ & $89(64)$ & $137(98,6)$ \\
\hline Pregunta 3 & $1(0,7)$ & $0(0)$ & $1(0,7)$ & $50(36)$ & $85(61,2)$ & $137(98,6)$ \\
\hline Formación & $\begin{array}{c}\mathbf{0} \\
\mathbf{n}(\%)\end{array}$ & $\begin{array}{c}1 \\
\text { n }(\%)\end{array}$ & $\begin{array}{c}2 \\
\text { n }(\%)\end{array}$ & $\begin{array}{c}3 \\
\text { n }(\%)\end{array}$ & $\begin{array}{c}4 \\
\text { n (\%) }\end{array}$ & $\begin{array}{l}\text { Total } \\
\text { n (\%) }\end{array}$ \\
\hline Pregunta 1 & $28(20,1)$ & $63(45,3)$ & $40(28,8)$ & $1(0,7)$ & $5(3,6)$ & $137(98,6)$ \\
\hline Pregunta 2 & $94(67,6)$ & $27(19,4)$ & $6(4,3)$ & $4(2,9)$ & $6(4,3)$ & $137(98,6)$ \\
\hline Pregunta 3 & $84(60,4)$ & $41(29,5)$ & $9(6,5)$ & $3(2,2)$ & $0(0)$ & $137(98,6)$ \\
\hline
\end{tabular}

tados de centros de salud o consultorios con industrias en su zona de influencia $(\mathrm{p}=0,026)$, entre los que tienen un contrato temporal $(\mathrm{p}=0,001)$ y entre los trabajadores del servicio de urgencias hospitalario $(\mathrm{p}=0,013)$. Los resultados completos están recogidos en la tabla 1 .

$\mathrm{Si}$ atendemos a los resultados obtenidos en la dimensión de habilidades prácticas, han obtenido puntuaciones significativamente mejores los médicos $(\mathrm{p}=0,006)$, los profesionales con industrias en su zona de trabajo ( $\mathrm{p}=0,031)$, los trabajadores con contrato temporal $(\mathrm{p}=0,027)$ y los profesionales del servicio de urgencias hospitalario $(\mathrm{p}=0,000)$. Los resultados completos de esta dimensión los podemos ver en la tabla 2.

En la dimensión que trata de medir las actitudes del personal sanitario ante el riesgo industrial y su predisposición a recibir formación y/o información sobre el mismo, las puntuaciones obtenidas han sido significativamente más altas entre las mujeres ( $\mathrm{p}=$ $0,036)$ y entre los trabajadores con contrato indefinido ( $\mathrm{p}=0,037)$; además se encontró una relación inversa y significativa entre las puntuaciones obtenidas y la edad ( $\mathrm{r}=-0,232$; $\mathrm{p}=0,000)$ y los años trabajados en el área $(\mathrm{r}=-$ $0,234 ; \mathrm{p}=0,006)$. En el caso de la primera pregunta sobre la percepción del riesgo 
industrial del área, la media obtenida es de 2,71 con una desviación estándar de 0,94 y un rango de 0 a 4 , siendo las puntuaciones significativamente mayores entre los trabajadores del centro de salud de Villalegre $(\mathrm{p}=0,037)$ y existiendo una correlación inversa y significativa $(\mathrm{r}=-0,293 ; \mathrm{p}=0,001)$ entre la puntuación obtenida y la edad de los encuestados. En la tabla 2 podemos ver los resultados completos de la dimensión actitudes.

En el caso de la formación de los profesionales sanitarios en el campo de las emergen- cias, han obtenido resultados significativamente mejores los profesionales de centros de salud o consultorios con industrias en su zona ( $\mathrm{p}=0,021)$, los trabajadores con contratos indefinidos $(\mathrm{p}=0,009)$ y los del servicio de urgencias hospitalario $(\mathrm{p}=0,000)$. También se ha encontrado una relación directa y significativa entre la puntuación en esta dimensión y la obtenida en conocimientos técnicos $(\mathrm{r}=0,635 ; \mathrm{p}=0,000)$, conocimientos del área $(\mathrm{r}=0,419 ; \mathrm{p}=0,000)$, habilidades prácticas $(\mathrm{r}=0,574 ; \mathrm{p}=0,000)$ y actitudes $(\mathrm{r}=0,259 ; \mathrm{p}=0,002)$. Los resultados de esta dimensión están recogidos en la tabla 2.

Tabla 3

Diferencias significativas I

\begin{tabular}{|c|c|c|c|c|c|}
\hline \multicolumn{6}{|c|}{ Diferencias entre médicos y enfermeras/os } \\
\hline Variable & Grupo & $\mathrm{N}$ & $\mu()$ & $\mathrm{F}(\mathrm{p})$ & $\mathrm{t}(\mathrm{p})$ \\
\hline Habilidades & Médicos & 64 & $5,26(3,32)$ & & \\
\hline prácticas & Enfermeras/os & 75 & $3,88(2,47)$ & $6,20(0,014)$ & $2,81(0,006)$ \\
\hline Pregunta 5 & Médicos & 64 & $1,09(0,71)$ & & \\
\hline Conocimientos & Enfermeras/os & 75 & $0,79(0,60)$ & $0,12(0,720)$ & $2,77(0,006)$ \\
\hline Pregunta 3 & Médicos & 64 & $1,31(1,15)$ & & \\
\hline Habilidades & Enfermeras/os & 75 & $0,76(0,77)$ & $15,59(0,000)$ & $3,36(0,001)$ \\
\hline Pregunta 5 & Médicos & 63 & $1,29(0,87)$ & & \\
\hline Habilidades & Enfermeras/os & 74 & $0,81(0,63)$ & $4,72(0,031)$ & $3,68(0,000)$ \\
\hline \multicolumn{6}{|c|}{ Diferencias entre hombres y mujeres } \\
\hline Variable & Grupo & $\mathrm{N}$ & $\mu()$ & $\mathrm{F}(\mathrm{p})$ & $\mathrm{t}(\mathrm{p})$ \\
\hline & Hombres & 55 & $9,25(2,28)$ & & \\
\hline Actitudes & Mujeres & 84 & $10,03(1,81)$ & $3,99(0,048)$ & $-2,13(0,036)$ \\
\hline Pregunta 5 & Hombres & 55 & $1,22(0,81)$ & & \\
\hline Habilidades & Mujeres & 82 & $0,90(0,75)$ & $0,10(0,747)$ & $2,34(0,021)$ \\
\hline Pregunta 3 & Hombres & 54 & $3,46(0,54)$ & & \\
\hline Actitudes & Mujeres & 83 & $3,67(0.61)$ & $0,98(0,324)$ & $-2,13(0,035)$ \\
\hline
\end{tabular}


Tabla 4

Diferencias significativas II

\begin{tabular}{|c|c|c|c|c|}
\hline \multirow{2}{*}{ Variable } & \multicolumn{3}{|c|}{ Diferencias entre tipos de contrato } & \multirow{2}{*}{ ANOVA } \\
\hline & Grupo & $\mathbf{N}$ & $\mu()$ & \\
\hline \multirow{4}{*}{$\begin{array}{c}\text { Conocimientos } \\
\text { técnicos }\end{array}$} & & & & $\mathrm{F}(\mathrm{p})$ \\
\hline & Fijo & 84 & $8,27(3,83)$ & \\
\hline & Indefinido & 32 & $10,12(4,49)$ & $5,92(0,003)$ \\
\hline & Temporal & 23 & $11,04(2,91)$ & \\
\hline \multirow{3}{*}{$\begin{array}{c}\text { Conocimientos del } \\
\text { área }\end{array}$} & Fijo & 84 & $3,61(3,58)$ & \multirow{3}{*}{$7,19(0,001)$} \\
\hline & Indefinido & 32 & $5,15(4,08)$ & \\
\hline & Temporal & 23 & $6,69(3,11)$ & \\
\hline \multirow{3}{*}{$\begin{array}{l}\text { Habilidades } \\
\text { prácticas }\end{array}$} & Fijo & 84 & $4,00(2,85)$ & \multirow{3}{*}{$3,71(0,027)$} \\
\hline & Indefinido & 32 & $5,03(3,37)$ & \\
\hline & Temporal & 23 & $5,69(2,34)$ & \\
\hline \multirow{3}{*}{ Actitudes } & Fijo & 84 & $9,36(2,24)$ & \multirow{3}{*}{$3,36(0,037)$} \\
\hline & Indefinido & 32 & $10,31(1,63)$ & \\
\hline & Temporal & 23 & $10,21(1,47)$ & \\
\hline \multirow{3}{*}{ Formación } & Fijo & 84 & $1,85(1,86)$ & \multirow{3}{*}{$4,90(0,009)$} \\
\hline & Indefinido & 32 & $3,21(2,74)$ & \\
\hline & Temporal & 23 & $2,17(1,85)$ & \\
\hline \multirow{3}{*}{$\begin{array}{c}\text { Pregunta } 5 \\
\text { Conocimientos }\end{array}$} & Fijo & 84 & $0,79(0,60)$ & \multirow{3}{*}{$5,81(0,004)$} \\
\hline & Indefinido & 32 & $1,06(0,80)$ & \\
\hline & Temporal & 23 & $1,26(0,54)$ & \\
\hline \multirow{3}{*}{$\begin{array}{c}\text { Pregunta } 7 \\
\text { Conocimientos } \\
\end{array}$} & Fijo & 82 & $1,61(0,97)$ & \multirow{3}{*}{$5,11(0,007)$} \\
\hline & Indefinido & 32 & $1,97(1,00)$ & \\
\hline & Temporal & 23 & $2,30(0,97)$ & \\
\hline \multirow{3}{*}{$\begin{array}{l}\text { Pregunta } 2 \\
\text { Formación }\end{array}$} & Fijo & 82 & $0,41(0,89)$ & \multirow{3}{*}{$3,17(0,045)$} \\
\hline & Indefinido & 32 & $0,94(1.37)$ & \\
\hline & Temporal & 23 & $0,48(0.79)$ & \\
\hline
\end{tabular}

Figura 1

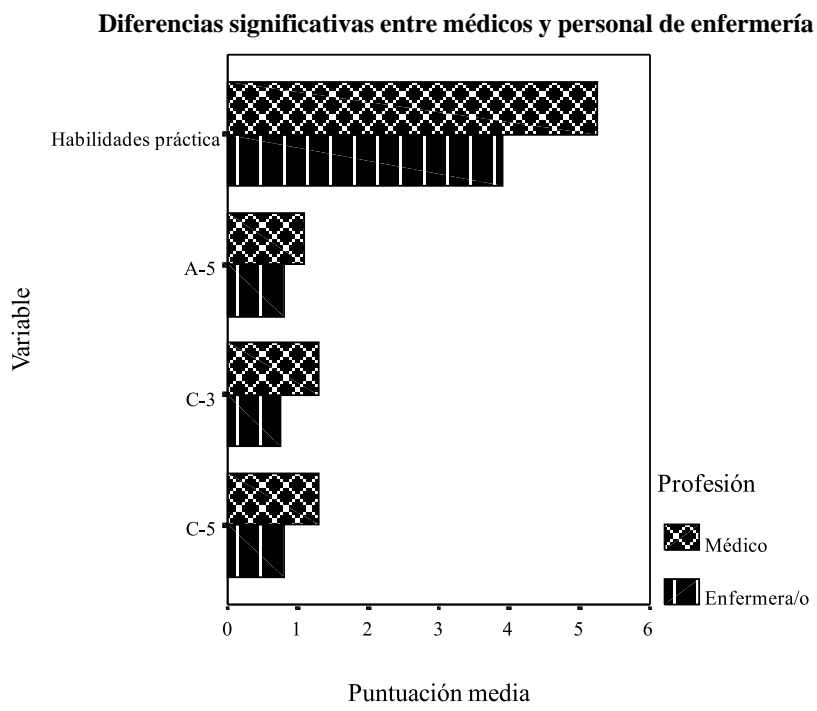


Tabla 5

Diferencias significativas III

\begin{tabular}{|c|c|c|c|c|}
\hline \multirow[b]{2}{*}{ Variable } & \multicolumn{3}{|c|}{ Diferencias entre centros de trabajo } & \multirow[b]{2}{*}{ ANOVA } \\
\hline & $\begin{array}{c}\text { Centro con } \\
\text { mejor puntuación }\end{array}$ & $\mathbf{N}$ & $\mu()$ & \\
\hline $\begin{array}{c}\text { Conocimientos } \\
\text { técnicos }\end{array}$ & urg & 27 & $13,25(4,50)$ & $\begin{array}{c}\mathrm{F}(\mathrm{p}) \\
5,02(0,000)\end{array}$ \\
\hline $\begin{array}{c}\text { Conocimientos del } \\
\text { área }\end{array}$ & urg & 27 & $7,00(4,47)$ & $2,05(0,013)$ \\
\hline $\begin{array}{l}\text { Habilidades } \\
\text { prácticas }\end{array}$ & urg & 27 & $7,33(3,67)$ & $3,39(0,000)$ \\
\hline Formación & urg & 27 & $4,44(3,09)$ & $3,17(0,000)$ \\
\hline $\begin{array}{c}\text { Pregunta } 3 \\
\text { Conocimientos } \\
\end{array}$ & urg & 27 & $2,56(0,85)$ & $7,69(0,000)$ \\
\hline $\begin{array}{c}\text { Pregunta } 6 \\
\text { Conocimientos }\end{array}$ & urg & 27 & $3,30(0,78)$ & $3,33(0,000)$ \\
\hline $\begin{array}{c}\text { Pregunta } 7 \\
\text { Conocimientos } \\
\end{array}$ & urg & 27 & $2,81(0,94)$ & $3,04(0,000)$ \\
\hline $\begin{array}{c}\text { Pregunta } 1 \\
\text { Conocimientos del } \\
\text { Área }\end{array}$ & urg & 26 & $2,31(1,38)$ & $2,00(0,016)$ \\
\hline $\begin{array}{l}\text { Pregunta } 3 \\
\text { Habilidades }\end{array}$ & urg & 27 & $2,19(1,00)$ & $6,74(0,000)$ \\
\hline $\begin{array}{l}\text { Pregunta } 5 \\
\text { Habilidades }\end{array}$ & urg & 26 & $1,65(0,98)$ & $2,26(0,006)$ \\
\hline $\begin{array}{l}\text { Pregunta } 1 \\
\text { Actitudes }\end{array}$ & 341 & 13 & $2,92(0,76)$ & $1,78(0,037)$ \\
\hline $\begin{array}{l}\text { Pregunta } 1 \\
\text { Formación }\end{array}$ & urg & 27 & $1,93(1,24)$ & $2,55(0,002)$ \\
\hline $\begin{array}{l}\text { Pregunta } 2 \\
\text { Formación } \\
\end{array}$ & urg & 27 & $1,59(1,53)$ & $2,80(0,001)$ \\
\hline
\end{tabular}

Figura 2

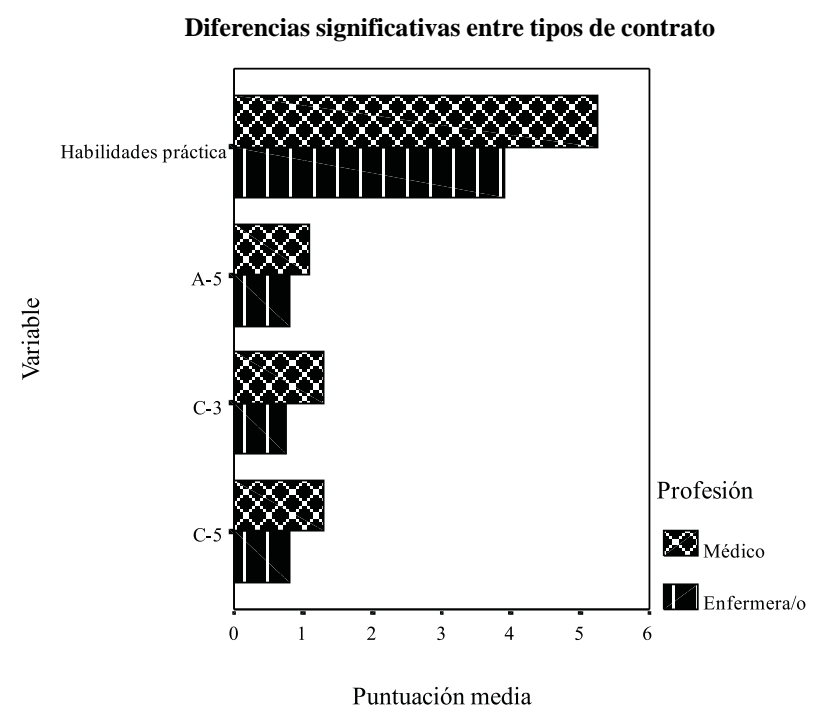


Figura 3

Representación del análisis de correlación bivariable

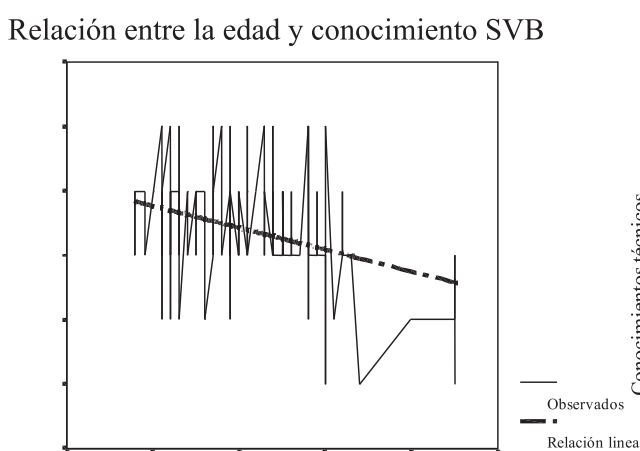

Edad

Relación entre formación y

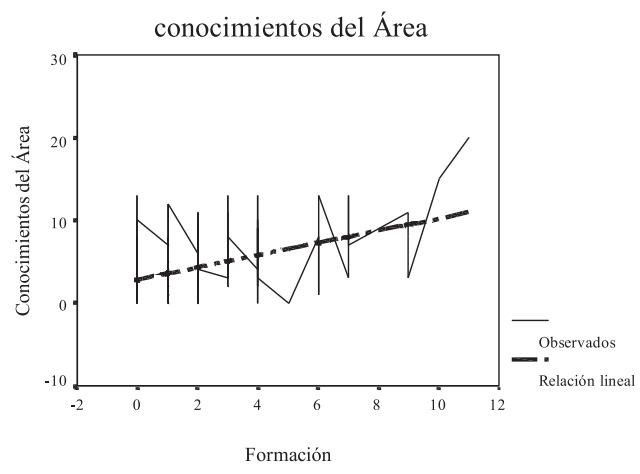

Los resultados entre los que se han encontrado diferencias estadísticamente significativas los podemos ver en las tablas 3,4 y 5 , quedando representados algunos
Relación entre formación y

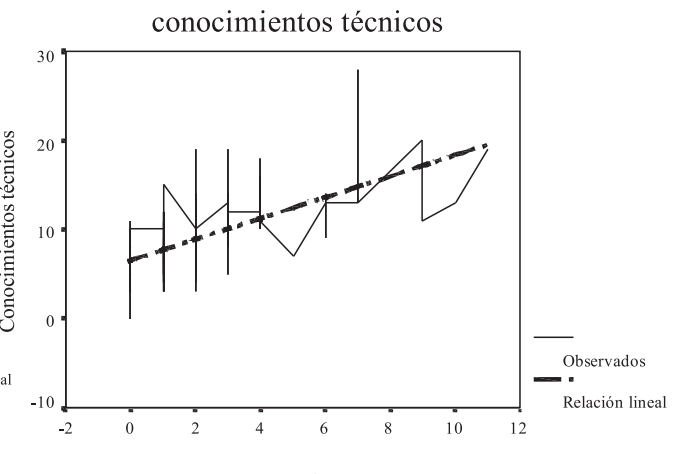

Formación

Relación entre formación y

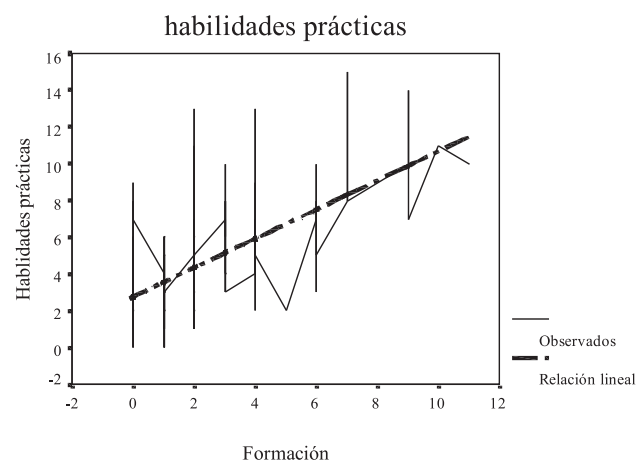

de ellos en las figuras 1 y 2 . En la tabla 6 queda recogido el análisis de correlación, quedando representado en parte en la figura 3. 
Tabla 6

Análisis de correlación

\begin{tabular}{|cc|}
\hline \multicolumn{1}{|c|}{ Análisis de correlación } \\
Variables & $\mathbf{r}(\mathbf{p})$ \\
\hline Años en área/ Actitudes & $-0,234(0,006)$ \\
Edad/ Conocimientos técnicos & $-0,232(0,007)$ \\
Edad/ Actitudes & $-0,399(0,000)$ \\
Años en área/ Pregunta 2 Actitudes & $-0,265(0,002)$ \\
Años en urgencias/ Pregunta 5 Habilidades & $0,235(0,007)$ \\
Edad/ Pregunta 3 Conocimientos & $-0,183(0,034)$ \\
Edad/ Pregunta 6 Conocimientos & $-0,299(0,000)$ \\
Edad/ Pregunta 7 Conocimientos & $-0,272(0,002)$ \\
Edad/ Pregunta 1 Actitudes & $-0,293(0,001)$ \\
Edad/ Pregunta 2 Actitudes & $-0,317(0,000)$ \\
Edad/ Pregunta 3 Actitudes & $-0,266(0,002)$ \\
Edad/ Pregunta 1 Formación & $-0,259(0,003)$ \\
Formación/ Conocimientos técnicos & $0,635(0,000)$ \\
Formación/ Conocimientos del Área & $0,419(0,000)$ \\
Formación/ Habilidades prácticas & $0,574(0,000)$ \\
Formación/ Actitudes & $0,259(0,002)$ \\
&
\end{tabular}

\section{DISCUSIÓN}

La consistencia interna de cada una de las dimensiones que se tratan de medir con el cuestionario era aceptable dado que el valor del coeficiente alfa de Cronbach era en casi todas ellas mayor de $0,7 \mathrm{y}$, en dos de ellas, muy próximo.

Los resultados globales de las encuestas muestran abundantes contestaciones con nulo o escaso, lo que denota pobres resultados en cuanto a la percepción que tienen los profesionales sanitarios del Área sobre su preparación para responder a un eventual desastre industrial y cuyas causas analizare- mos más adelante. Además, probablemente los resultados reales sean peores que los obtenidos al ser razonable esperar que las personas con un interés especial por el campo de las emergencias médicas o el riesgo industrial tenderán a responder más y mejor a un cuestionario como el realizado, mientras que en los profesionales con menores conocimientos e interés el índice de respuesta será menor.

Con respecto a la dimensión que trata de medir los conocimientos técnicos, sería interesante explicar que el hecho de que la puntuación obtenida sea significativamente mayor entre las personas con contratos tem- 
porales podría ser debido a que se ha encontrado una relación estadísticamente significativa ( $\mathrm{p}=0,000)$ entre el tipo de contrato y la edad, dándose más los contratos temporales entre las personas jóvenes. Tras realizar un análisis estratificado por edad de las puntuaciones obtenidas en esta dimensión, la influencia del tipo de contrato es mayor en el grupo de edad de 28 a 40 años $(\mathrm{p}=0,031)$. También han sido las puntuaciones significativamente mayores entre el personal del servicio de urgencias hospitalario, algo que sería esperable, aunque en este caso también se ha encontrado que el personal del este servicio tiene una edad menor que la media de todos los encuestados $(\mathrm{p}=0,000)$. Si analizamos los resultados de cada una de las preguntas de esta dimensión, consideramos que es de vital importancia el que los profesionales sanitarios que acudan al lugar de un accidente con múltiples víctimas, sea del tipo que sea, tengan un conocimiento al menos suficiente sobre el triage, y es destacable el que más del $60 \%$ de los encuestados no alcancen este nivel, e incluso algunos desconozcan el término. Son incluso más pobres los resultados obtenidos en las preguntas que valoran específicamente el conocimiento sobre la descontaminación ${ }^{25}$ y tratamiento de pacientes, algo que se podría mejorar con cursos cortos sobre el tema, sobre todo con respecto a la descontaminación, ya que creemos que unos conocimientos básicos sobre ésta se podrían alcanzar muy rápido. Aunque aparentemente los resultados sobre el conocimiento de las maniobras de soporte vital básico son bastante mejores que los obtenidos en otras preguntas (sobre todo las que hacen referencia a las placas de identificación de sustancias peligrosas y a los equipos de protección, consideradas como muy específicas), creemos que el $100 \%$ de los profesionales sanitarios debería de tener al menos un conocimiento bueno o muy bueno sobre las mismas, ya que consideramos que el conocimiento suficiente debería de tenerlo toda la población general. Nos parece preocupante el que más de la mitad de los encuestados no considere que su nivel sea bueno o muy bueno, y más aún el que el 10\% lo considere nulo o escaso. Ya que las maniobras de soporte vital básico no es algo que entre en el trabajo diario de la mayoría de los profesionales sanitarios, creemos que se deberían de llevar a cabo cursos de reciclaje en la materia con una periodicidad determinada. Con respecto a las maniobras de soporte vital avanzado, como era de esperar los resultados son peores que en el caso anterior, sobre todo por ser algo más específico, aunque de todas formas no es excusable el desconocimiento de las mismas por el $4.3 \%$ de los encuestados ni el que poco más de la mitad tengan un conocimiento suficiente o mejor. Creemos que la gran mayoría debería de tener al menos un conocimiento suficiente, y lo dicho en el caso anterior con respecto a la formación continuada se podría aplicar en este caso. La mayoría de los encuestados $(48,2 \%)$ asocian el término catástrofe al hecho de que haya un gran número de afectados. Esta quizás sea una manera indirecta de decir que los recursos se vean desbordados, ya que seguramente por sus experiencias profesionales así lo han visto, es decir, si se tiene un gran número de afectados probablemente los recursos se vean desbordados en algún momento, aunque esto no siempre es así. En el caso de esta pregunta la respuesta que sería correcta según la OMS sería el que hablamos de una catástrofe cuando los recursos están desbordados, y consideramos escaso el número de encuestados que así lo indican, lo que puede ser interpretado como una falta de formación básica en el campo de los desastres en general.

En el caso de los conocimientos del Área, la puntuación obtenida ha sido significativamente mayor entre los profesionales con contratos temporales. La explicación que podría tener este hecho es que estos profesionales, al tener contratos temporales, suelen trabajar en prácticamente todas las zonas básicas de salud del Área, por lo que conocen las mismas de una manera más completa que una persona que lleve mucho tiempo en el mismo puesto de trabajo, lo que le propor- 
cionará un conocimiento amplio de su zona de trabajo pero no del resto. También tienen un conocimiento del Área significativamente mejor los trabajadores del servicio de urgencias hospitalario, lo que podría ser debido a que una gran mayoría de estos antes de desempeñar sus tareas en dicho servicio han tenido contratos temporales en Atención Primaria, por lo que ocurriría lo mismo que en el caso anterior. En general, los resultados de esta dimensión son peores que en el caso anterior, lo que demuestra una falta de información a los profesionales implicados en la respuesta sanitaria ante un desastre industrial.

Si tratamos de analizar los resultados obtenidos en la dimensión de las habilidades prácticas, aquella que trata de medir cómo realizaría el profesional sanitario sus funciones en el lugar de un accidente con sustancias peligrosas, estos son, como en los casos anteriores, bastante pobres. En este caso hemos encontrado puntuaciones significativamente mayores entre los profesionales del servicio de urgencias hospitalario; esto era esperable por ser los profesionales que día a día se enfrentan a situaciones clínicas urgentes, por lo que realmente es su trabajo diario, al contrario que los profesionales de Atención Primaria, en cuyo trabajo la atención a patologías urgentes no tiene tanto peso como en los anteriores. También han obtenido puntuaciones significativamente mayores los médicos, lo que podría implicar y justificar el que sobre estos recayese la responsabilidad ante las distintas situaciones urgentes que se pueden dar en el ámbito industrial, así como los encuestados con contrato temporal, lo que podría ser debido a una mejora en la formación de los profesionales sanitarios en los últimos tiempos.

En el caso de la dimensión que trata de medir las actitudes del personal sanitario ante el riesgo de desastre industrial, el hecho de que las mujeres, los trabajadores con contrato indefinido, los que llevan menos años en el Área y los más jóvenes hayan obtenido puntuaciones significativamente más altas que el resto de los grupos significa que los anteriormente mencionados tienen una percepción subjetiva de riesgo industrial mayor así como que tienen una predisposición mayor a recibir información sobre el riesgo industrial del Área y a recibir formación. De estos factores, creemos que el más determinante es la edad, ya que hemos encontrado que las mujeres encuestadas son más jóvenes $(\mathrm{p}=0,000)$ y que entre la edad y los años trabajados en el Ârea existe una correlación directa y significativa $(\mathrm{r}=0,618 ; \mathrm{p}=0,000)$. Analizando los resultados globales de las preguntas de esta dimensión, vemos que hay una tendencia a una percepción del riesgo como medio-alto, atendiendo a la puntuación media obtenida. Esto nos da a entender que realmente los profesionales sanitarios son conscientes del riesgo que conlleva la actividad industrial, sin entrar a discutir sobre el hecho de asumirlo o no, y sobre todo teniendo en cuenta el que por ser una percepción ésta es totalmente subjetiva y por tanto dependiente de muchos factores socioculturales como lugar de residencia, tiempo que se lleva viviendo en la zona o relación familiar con las industrias, factores que no vamos a analizar en este caso. Sí hemos comprobado que existe una relación inversa y significativa entre esta percepción del riesgo y la edad de los encuestados, algo que puede explicarse por una sensibilización mayor de las generaciones jóvenes ante la problemática medio ambiental. Probablemente conscientes de este hecho, el interés general en recibir conocimientos básicos sobre cómo actuar ante un desastre industrial es bastante alto si atendemos a los resultados. Bien es cierto que quizás los mismos pueden sobreestimar este interés debido a que por el simple hecho de responder al cuestionario ya se está demostrando un interés determinado; aún así, los resultados obtenidos en este caso son tan significativos a favor de recibir dicha formación que no creemos que si hubiesen contestado el $100 \%$ del universo estadístico hubiese un cambio de tendencia importante en los resultados. Lo mismo sucede con el 
interés en recibir información sobre el riesgo que suponen las industrias asentadas en su Ârea; hay una predisposición totalmente clara a ser informados sobre el mismo, con las salvedades indicadas en el caso anterior. En estos dos últimos casos la relación inversa y significativa encontrada entre el interés en ser formados e informados y la edad de los encuestados nos da a entender una mayor inquietud por parte de los profesionales más jóvenes en adquirir nuevos conocimientos.

En cuanto al apartado de formación se refiere, la formación en emergencias es prácticamente nula para la mayoría de los encuestados. Este hecho, que quizás no fuese importante en otras zonas, en el caso concreto del Área III creemos que es bastante significativo por ser una zona geográfica con concentración de riesgos susceptibles de poder provocar un desastre, y creemos que sería deseable que al menos los profesionales sanitarios de un Área con riesgos potenciales recibiesen formación básica sobre sus actuaciones en caso de desastre. Como era de esperar, la puntuación total obtenida en esta dimensión ha sido significativamente mayor entre los profesionales del servicio de urgencias hospitalario, ya que su formación continuada está básicamente enfocada hacia las distintas facetas de las urgencias y emergencias médicas. También la puntuación es significativamente mayor entre los trabajadores con contratos indefinidos; algo que puede ser debido a que han tenido el suficiente tiempo como para realizar un importante número de cursos. En cuanto a los resultados en cada pregunta, un porcentaje bastante considerable de encuestados ha realizado en los últimos 2 años uno o más cursos de RCP básica y/o avanzada, aunque sería deseable que la práctica totalidad de los profesionales sanitarios realizasen cursos de reciclaje en dicha materia por ser algo de obligado conocimiento por su parte en cualquier faceta de su profesión.

Se puede concluir, a la vista de los resultados obtenidos que existe un déficit de infor- mación y de formación en los profesionales sanitarios, tanto en aspectos básicos como específicos de la repuesta ante desastres industriales, que contrasta con el interés manifestado en adquirir dichos conocimientos. Esta situación resulta paradójica en un área sanitaria con el perfil de riesgo industrial hallado. Por otro lado, las carencias detectadas son consecuencia del déficit de flujos de información entre los distintos estamentos relacionados en la respuesta ante desastres industriales, lo que demuestra fallos en la fase de implantación de los planes de emergencia exterior entre el personal sanitario del Ârea.

Consideramos que es necesario establecer un programa básico de formación e información a los profesionales sanitarios en materia de riesgo de emergencia industrial, en las vertientes de prevención, preparación y respuesta sanitaria ante desastres industriales, adaptado específicamente a los peligros detectados. El énfasis del programa deberá ponerse en la capacitación para realizar maniobras de reanimación cardiopulmonar, organización de la asistencia sanitaria en el lugar del desastre, triage y descontaminación de pacientes. Es necesario, asimismo, definir las funciones de los distintos equipos de asistencia sanitaria (Hospital, UVI móvil, Atención Primaria) de manera que cada uno de ellos desarrolle al máximo sus posibilidades de respuesta ante un desastre industrial.

\section{BIBLIOGRAFÍA}

1. Lillibridge SR, Noji EK, Burkle FM. Disaster assessment: the emergency evaluation of a population affected by a disaster. Ann Emerg Med 1993; 22: $1715-1720$.

2. Bhopal Working Group. The public health implications of the Bhopal disaster. Am J Pub Health 1987; 77: $230-236$.

3. Morrow BH. Identifying and mapping community vulnerability. Disasters 1999; 23: 1-18.

4. Waeckerle JF, Lillibridge SR, Noji EK, Burkle FM. Disaster Medicine: Challenges for today. Ann Emerg Med 1994; 23: 715-718. 
5. Castro R, Arcos P. El riesgo de desastre químico como cuestión de salud pública. Rev Esp Salud Pública 1998; 72: 481-500.

6. Wheeler H. Major incident planning particularly those including chemicals. Emerg Nurse 1998; 6: $12-16$

7. Waeckerle JF. Disaster Planning and Response. N Engl J Med 1991; 324: 815-21.

8. Rodgers JC. Chemical incident plannning: a review of the literature. Accident and Emergency Nursing 1998; 6: 155-9.

9. Organisation for Economic Cooperation and Development. Guidance concerning Health Aspects of Chemical Accidents. Paris, 1996.

10. Agency for Toxic Substances and Disease Registry, United States Department of Health and Human Services, Public Health Services. Hospital emergency departments: a planning guide for the management of contaminated patients (Managing Hazardous Substances Incidents, Vol.1,2,3). Atlanta: Agency for Toxic Substances and Disease Registry; 1992.

11. Sociedad Asturiana de Estudios Económicos e Industriales (SADEI). Nomenclator de Entidades de Población de Asturias 1996. Avilés: SADEI; 1997.

12. Boletín Oficial del Principado de Asturias. Decreto 112/1984, de 6 de Septiembre, por el que se aprueba, con carácter definitivo, el Mapa Sanitario de Asturias, y se dictan normas para su puesta en práctica. BOPA núm. 232, 6-10-1984 (corrección de errores núm. 280, 4-12-1984)

13. Castro R, Arcos P, del Busto F, Cuartas T. Diagnóstico del riesgo industrial desde la perspectiva de salud pública. X Congreso de la sociedad española de medicina preventiva y salud pública e higiene. Libro de actas y abstracts: pág. 157-168.Gijón, Noviembre 1999.

14. Castro R, Arcos P. El perfil de riesgo químico del Área Sanitaria III de Asturias. III Forum Europeo de Ciencia, Seguridad e Higiene. Libro de ponencias y comunicaciones: pág. 87.Oviedo, Septiembre 1998.
15. Arcos González P., González Carril F., Huerta González M, Cueto Espinar A. El concepto de desastre y su aplicación en Asturias. Rev San Hig Pública 1994; 68: 573-578.

16. Somers GT, Drinkwater EJ, Torcello N. The general practitioner as first responder in a major medical emergency. Aust Fam Physician 1997; 26: 1406-9.

17. Somers GT, Maxfield N, Drinkwater EJ. General practitioner preparedness to respond to a medical disaster part I: skills and equipment. Aust Fam Physician 1999; 28 (Suppl 1): 3-9.

18. Somers GT, Maxfield N, Drinkwater EJ. General practitioner preparedness to respond to a medical disaster part II: ability and training. Aust Fam Physician 1999; 28 (Suppl 1): 10-14

19. Mark AK, Cisek J, Rose SR. Respuesta del servicio de urgencia a los accidentes con sustancias peligrosas. Clínicas de medicina de urgencia de Norteamérica (edición española) 1994; 2: 489-513.

20. Tur-Kaspa I, Lev EI, Hendler I, Siebner R, Shapira Y, Shemer J. Preparing hospitals for toxicological mass casualties events. Cri Care Med 1999; 27: 1004-1008.

21. Totenhofer RI, Kierce M. It's a disaster: emergency departments preparation for a chemical incident or disaster. Accid Emerg Nurs 1999; 7: 141-147.

22. Boletín Oficial del Estado. Real Decreto 886/1988, de 15 de julio, sobre prevención de accidentes mayores en determinadas actividades industriales. BOE núm. 187, 5-8-1988.

23. Boletín Oficial del Estado. Real Decreto $1254 / 1999$, de 16 de julio, por el que se aprueban las medidas de control de los riesgos inherentes a los accidentes graves en los que intervengan sustancias peligrosas. núm. 172, 20-7-1999.

24. Dunn G. Design and analysis of reliability studies the statistical evolution of measurement errors. 1a edición. New York: Oxford University Press; 1989.

25. Cox RD. Decontamination and Management of Hazardous Materials Exposure Victims in the Emergency Department. Ann Emerg Med 1994; 23: 761-770. 
Anexo I

Cuesitonario

\section{Instrucciones}

1. El objetivo de este estudio es conocer el grado de preparación y las actitudes del personal sanitario del Área III para responder ante un potencial desastre de tipo industrial.

2. Lea cuidadosamente las preguntas y trate de contestarlas todas. La encuesta es anónima.

3. El cuestionario consta de 28 preguntas, que podrá contestar en unos 10-15 minutos totales.

4. Los resultados servirán para detectar posibles deficiencias y, en su caso, proponer medidas de mejora.

5. Le agradecemos su participación en el presente estudio y el tiempo dedicado.

\section{Código cuestionario}

Año de nacimiento

Sexo

Categoría profesional (solo una):

1. Médico/a

2. Médico/a con formación MIR

3. Médico/a coordinador/a

4. Médico/a coordinador/a con formación MIR

5. ATS/DUE

6. ATS/DUE coordinador/a

Tipo de contrato: Indefinido - Fijo - Temporal

Años trabajados en este Área de Salud

Años trabajados en asistencia sanitaria urgente (incluyendo guardias de atención primaria)

Lugar de trabajo (marque con una $\mathrm{x}$ )

1. ZBS 3.1: 3.1.1: C.S. Sabugo

2. ZBS 3.2: 3.2.1: Ambulatorio Central de Avilés 3.2.2: Servicio normal de urgencias

3. ZBS 3.3: 3.3.1: C.S. La Magdalena 3.3.2: Consultorio La Carriona 3.3.3: Consultorio de Illas

4. ZBS 3.4: 3.4.1: C.S. de Villalegre 3.4.2: Consultorio de Llaranes

5. ZBS 3.5: 3.5.1: C.S. Las Vegas 3.5.2: Consultorio de Trasona 3.5.3: Consultorio de Cancienes

6. ZBS 3.6: 3.6.1: C.S. de Cudillero 3.6.2: Consultorio San Martín de Luiña

7. ZBS 3.7: 3.7.1: C.S. de Pravia 3.7.2: Consultorio de Muros del Nalón 3.7.3: Consultorio de Soto del Barco 3.7.4: Consultorio de San Juan de la Arena 3.7.5: Consultorio de San Esteban de Pravia. 
8. ZBS 3.8: 3.8.1: C.S. de Castrillón 3.8.2: Consultorio de Raíces

9. ZBS 3.9: 3.9.1: C.S. de Luanco 3.9.2: Consultorio de San Jorge de Manzaneda

Servicio de Urgencias Hospital San Agustín

\section{A. Conocimientos técnicos}

1. Su conocimiento sobre las placas o símbolos de identificación de sustancias peligrosas considera que es: Nulo 0; Escaso 1; Suficiente 2; Bueno 3; Muy bueno 4; desconozco el término.

2. Su conocimiento sobre los equipos de protección contra sustancias peligrosas considera que es:

Nulo 0; Escaso 1; Suficiente 2; Bueno 3; Muy bueno 4; desconozco el término.

3. Su conocimiento sobre el triage considera que es: Nulo 0; Escaso 1; Suficiente 2; Bueno 3; Muy bueno 4; desconozco el término.

4. Su conocimiento sobre las normas básicas de descontaminación de pacientes en un accidente con sustancias peligrosas considera que es: Nulo 0; Escaso 1; Suficiente 2; Bueno 3; Muy bueno 4; desconozco el término.

5. Su conocimiento sobre el tratamiento de los afectados en un accidente con sustancias peligrosas considera que es: Nulo 0; Escaso 1; Suficiente 2; Bueno 3; Muy bueno 4; desconozco el término.

6. Su conocimiento sobre las maniobras de RCP básica considera que es: Nulo 0; Escaso 1; Suficiente 2; Bueno 3; Muy bueno 4; desconozco el término.

7. Su conocimiento sobre la RCP avanzada considera que es: Nulo 0; Escaso 1; Suficiente 2; Bueno 3; Muy bueno 4; desconozco el término.

8. ¿Qué considera usted que es una catástrofe?

\section{B. Conocimientos del área}

1. ¿Sabe si hay algún Plan de Emergencia Sanitario de respuesta ante un accidente industrial en el Área III? No sé si lo hay (0) No, no lo hay (1) Creo que sí, pero lo desconozco (2) Sé que lo hay pero lo desconozco (3) Sí, lo hay y lo conozco (4).

2. ¿Su conocimiento acerca del papel que tendría usted dentro de un plan de emergencia de riesgo industrial considera que es > : Nulo 0; Escaso 1; Suficiente 2; Bueno 3; Muy bueno 4.

3. ¿Sabe si está usted incluido dentro de los recursos humanos adscritos a algún plan de emergencia exterior? No, no pertenezco (0); No estoy seguro (1); Sí, pertenezco (2).

4. Su grado de conocimiento de sustancias peligrosas y peligros en las industrias del Área III considera que es: Nulo 0; Escaso 1; Suficiente 2; Bueno 3; Muy bueno 4; desconozco el término.

5. ¿Qué tipo de catástrofe cree usted que es más probable que ocurra en el Área III? (Puede responder a más de una): Natural (inundación, etc.); Concentraciones humanas (conciertos, etc.); Accidente de tránsito con múltiples víctimas (tráfico, tren, avión); Transporte de mercancías peligrosas; Accidente mayor industrial. Otros (especifique cuál).

6. El número de empresas que tienen Plan de Emergencia Exterior en el Área III es: No lo sé 0; Ninguna 1;1 2;23; Más de 24. 
7. La información que ha recibido sobre sustancias peligrosas y peligros en el Área III considera que ha sido? Nulo, no he recibido ninguna (0)Escaso (1) Suficiente (2) Bueno (3) Muy bueno (4).

\section{Habilidades prácticas}

1. ¿Cómo diría que es su capacidad para informarse sobre un producto químico y sus características en caso de catástrofe industrial? Nula (0) Escasa (1) Suficiente (2) Buena(3) Muy buena (4).

2. Su habilidad en el uso de Equipos de Protección ante Riesgo Químico considera que es:

Nula (0) Escasa (1) Suficiente (2) Buena (3) Muy buena (4) (desconozco el término).

3. Su habilidad a la hora de realizar un triage en el lugar de un desastre industrial considera que es: Nula (0) Escasa (1) Suficiente (2) Buena (3) Muy buena (4) (desconozco el término).

4. Su habilidad a la hora de aplicar los Procedimientos Básicos de Descontaminación de afectados por sustancias peligrosas considera que es: Nula (0) Escasa (1)Suficiente (2) Buena (3) Muy buena (4) (desconozco el término).

5. Su capacidad para organizar la asistencia sanitaria en el lugar de un accidente con sustancias peligrosas considera que es: Nula (0) Escasa (1) Suficiente (2) Buena (3) Muy buena (4).

6. ¿Qué información facilitaría usted al Centro Coordinador de Emergencias si fuera la primera persona en llegar a un accidente con sustancias peligrosas?

\section{Actitudes}

1. Valore de 0 (riesgo nulo) a 4 (riesgo máximo) el grado de riesgo de accidente industrial que usted cree que tiene el Área III:

2. El personal sanitario del Área III debería recibir conocimientos básicos sobre cómo actuar ante un desastre industrial: Totalmente en desacuerdo 0; En desacuerdo 1; Indiferente 2; De acuerdo 3; Totalmente de acuerdo 4.

3. El personal sanitario debería de recibir información sobre el riesgo que suponen las industrias asentadas en su Área: Totalmente en desacuerdo 0; En desacuerdo 1; Indiferente 2; De acuerdo 3; Totalmente de acuerdo 4 .

\section{E. Formación}

1. Señale el número de Cursos de RCP básica y/o avanzada que ha realizado en los últimos 2 años.

Ninguno (0) 1 (1) 2 (2) 3 (3) Más de 3 (4).

2. Señale el número de Cursos de Emergencias que ha realizado en los que se haya tratado el tema de las catástrofes. Ninguno (0)1 (1) 2 (2) 3 (3) Más de 3 (4).

3. Señale el número de simulacros de catástrofe en los que ha participado como personal sanitario. Ninguno (0) 1 (1) 2 (2) 3 (3) Más de 3 (4). 